Cahiers $d u$ MONDE RUSSE

\section{Cahiers du monde russe}

Russie - Empire russe - Union soviétique et États indépendants

$58 / 4 \mid 2017$

Varia

\title{
Erika Wolf, Aleksandr Zhitomirsky: Photomontage as a Weapon of World War II and the Cold War
}

Juliette Milbach

\section{OpenEdition}

\section{Journals}

Édition électronique

URL : http://journals.openedition.org/monderusse/10203

DOI : $10.4000 /$ monderusse. 10203

ISSN : 1777-5388

\section{Éditeur}

Éditions de l'EHESS

\section{Édition imprimée}

Date de publication : 1 octobre 2017

Pagination : 762-764

ISBN : 978-2-7132-2698-4

ISSN : $1252-6576$

\section{Référence électronique}

Juliette Milbach, «Erika Wolf, Aleksandr Zhitomirsky: Photomontage as a Weapon of World War II and the Cold War », Cahiers du monde russe [En ligne], 58/4 | 2017, mis en ligne le 01 octobre 2017,

consulté le 06 janvier 2021. URL : http://journals.openedition.org/monderusse/10203 ; DOI : https:// doi.org/10.4000/monderusse. 10203 
Erika WOLF

\section{Aleksandr Zhitomirsky}

\section{Photomontage as a Weapon of World War II and the Cold War}

New Haven - London : The Art Institute of Chicago distributed by Yale University Press, 2016, 369 p., 300 ill. couleurs

En septembre 2016, l'Institut d'art de Chicago inaugurait une exposition, « Humanism + Dynamite $=$ The Soviet Photomontages of Aleksandr Zhitomirsky », dédiée à Aleksandr Žitomirskij (1907-1993), l'un des acteurs phares du photomontage, notamment pendant la Seconde Guerre mondiale, mais pas seulement. Pour accompagner cette exposition, Erika Wolf, professeur associée à l'université d'Ottago 
(Nouvelle-Zélande) et déjà auteur d'un ouvrage sur l'art de l'affiche (Koretsky: The Soviet photo poster : 1930-1984, New York, New Press, 2012), a livré non pas un catalogue, mais cette très belle et utile étude monographique d'un artiste méconnu, dont l'œuvre a été peu étudiée jusqu'à présent. Physiquement, l'ouvrage reproduit 120 photomontages, accompagnés de notices explicatives détaillées qui replacent cinq décennies de travail dans leur contexte de production et de diffusion. Un texte, divisé en quatre chapitres, retrace la carrière de Žitomirskij et analyse son œuvre. De plus, et il s'agit là d'un apport substantiel, Erika Wolf met à notre disposition un certain nombre de sources primaires de première importance traduites du russe. Les auteurs en sont Žitomirskij lui-même et John Heartfield (1881-1968), pionnier du photomontage allemand, avec lequel Žitomirskij entretint une longue amitié et dont l'œuvre ne fut pas sans inspirer la sienne. La traduction de larges extraits du seul livre publié de Žitomirskij, Iskusstvo političeskogo fotomontaža : Sovety hudožniku [The Art of Political Photomontage : Advice for the Artist] (Moscou : Plakat, 1983) constitue une riche source d'informations pour le lecteur. Par le truchement de cette présentation des sources primaires, Wolf apporte un réel éclairage sur les rapports entre les deux artistes. Les annexes sont complétées par une chronologie et une bibliographie.

Né à Rostov-sur-le-Don, Žitomirskij est le fils d'un pharmacien qui meurt du typhus pendant la guerre civile. Membre de la RABIS (Union des travailleurs artistiques) depuis 1923, il arrive à Moscou en 1925. Il travaille avec l'Association des artistes de la Russie révolutionnaire, groupe proche du pouvoir dans les années 1920. Il apprend également certaines techniques auprès de Vladimir Favorskij. Analysant à la fois les contraintes de production et l'iconographie, Erika Wolf décrit les travaux de Žitomirskij pour le magazine Metallist et pour Osoaviahim (les feuilles à large diffusion de la Société pour la promotion de la défense de l'aviation et de la chimie) et qualifie d'indépendante l'activité de Žitomirskij. Ce terme aurait sans doute mérité un développement plus approfondi dans le contexte particulier du stalinisme. Wolf présente l'année 1930 comme un tournant dans la carrière de Žitomirskij. En effet, celui-ci devient un artiste reconnu lorsqu'il publie une série de trois photomontages dans la prestigieuse revue Smena, qui emploie des artistes de premier ordre comme Aleksandr Dejneka et, plus tard, Aleksandr Rodčenko, Varvara Stepanova ou encore Boris Ignatovič. Dans les années 1930, l'artiste participe aussi à Stroim (qui sera plus ou moins remplacé par Illjustrirovannaja gazeta un peu plus tard). Il est également membre du comité éditorial de SSSR na stroike. En 1939, son talent est consacré dans le premier numéro de Industrija socializma. En reconstituant le début de sa carrière, Erika Wolf casse le mythe historiographique d'une naissance à la création exaltée par le sursaut patriotique qu'engendre la guerre, vision romantique de l'intention artistique qui, selon elle, avait été entretenue par des écrits et des propos d'Heartfield.

Cependant, compte-tenu de la nature de l'ouvrage, à la fois monographie et catalogue, l'étude de Wolf se présente avant tout comme une aide pour décrypter les images. Des analyses iconographiques permettent notamment à Wolf de déterminer les réemplois dans l'œuvre de Žitormirskij. À titre d'exemple, elle présente la 
couverture pour Smena de 1930, qui figure un conducteur de tracteur déjà présent sur la couverture du numéro de Mettalist d'octobre 1929. Exemple précoce de réutilisation, voire d'autocitation, dont Žitomirskij fera de plus en plus usage au fil du temps.

Erika Wolf fait état des difficultés rencontrées dans son travail, en particulier lorsqu'il s'agit d'authentifier avec certitude l'auteur de photomontages non signés. Dans son analyse d'images, Wolf insiste principalement sur la production et détermine en détail les images qui précèdent le photomontage. Elle note également la grande attention qui est portée à leur réception par le public contemporain, qu'il soit soviétique ou étranger, allemand et américain en particulier. L'étude révèle aussi les liens et les distances que Žitomirskij entretient avec l'œuvre de Gustav Klutsis bien sûr, mais aussi avec celle d'artistes aux pratiques artistiques plus éloignées de la sienne, comme El Lisickij. L'ensemble de ces analyses sont d'un apport décisif pour quiconque s'intéresse à la culture visuelle en Union soviétique.

Sans aller jusqu'à parler de réhabilitation, l'ambition de Wolf est de laisser entrevoir la plus grande complexité d'une production artistique, somme toute assez méprisée par les recherches occidentales sur le rapport compliqué de l'art soviétique officiel au photomontage. Elle souligne ainsi la grande qualité plastique de nombreuses images. Entre autres exemples, retenons simplement (dans le troisième chapitre, celui consacré à la Guerre froide) l'analyse du photomontage «Harry Truman : the Hysterical War Drummer », choisi pour illustrer la couverture de cet ouvrage et paru en mars 1948 dans Literaturnaia gazeta.

En présentant l'ensemble des thématiques traitées par Žitomirskij, l'ouvrage dévoile la grande richesse des sources d'inspiration de l'artiste. Il offre au lecteur une plongée dans la presse illustrée de la période soviétique, dont le fonctionnement et les lignes esthétiques sont parfaitement examinés. Un aspect, qui pourrait échapper au lecteur français, est relevé avec humour par James Rondeau, directeur de l'Institut d'Art de Chicago, dans sa préface à l'ouvrage : « It will be especially enlightning for American readers to learn the Soviet point of view on their country's actions around the world [...]». p. 7. Des problématiques qui trouvent donc parfaitement leur place dans le contemporain. Regrettons simplement que cette monographie à valeur de catalogue ne réserve qu'une place trop marginale (deux pages seulement, 92-94) aux autres activités de Žitomirskij, en particulier le dessin.

Enfin, n'omettons pas de mentionner que ce projet est redevable aux recherches menées dans la Ne Boltai Collection! qui, sauf indication contraire, détient le copyright de toutes les illustrations du livre. Cette collection pragoise permet de mettre en images plusieurs périodes soviétiques de la révolution à la Guerre froide, avec des ensembles substantiels consacrés aux fenêtres Rosta notamment. Certes, nous pourrions objecter l'embarras à tenir pour exhaustive une monographie établie sur la base d'une seule collection. Pourtant, et en dépit de ce qui pourrait apparaître comme une contrainte et un défaut, force est de constater que la monographie proposée par Erika Wolf retrace parfaitement l'ensemble de la carrière de Žitomirskij.

\section{Juliette Milbach}

